\section{Revista de Investigación en Logopedia}

ISSN: $1131-8635$

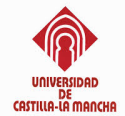

\title{
Manifestaciones lingüísticas en personas mayores: el papel de la intervención logopédica en el envejecimiento sano y patológico
}

\author{
Enrique González Martín; Nieves Mendizábal de la Cruz²; Natalia Jimeno Bulnes³; Carolina \\ Sánchez Gil ${ }^{4}$
}

Recibido: 27 de junio de 2018 / Revisado: 1 de octubre de 2018 / Aceptado: 31 de octubre de 2018

Resumen. En los últimos años ha cobrado especial relevancia incrementar los conocimientos acerca de las capacidades lingüístico-comunicativas de la población con una edad superior a 65 años. Los cambios biológicos, psicológicos y sociales que se producen en este grupo de personas deben ser atendidos teniendo en cuenta la interacción entre los dominios cognitivos y lingüísticos. El objetivo de este trabajo es realizar una revisión bibliográfica de las manifestaciones lingüísticas en personas mayores, tanto en el envejecimiento sano como patológico. Los resultados obtenidos indican que, en general, los rasgos más sobresalientes se manifiestan principalmente en la dificultad de acceso a la etiqueta léxica de las palabras, el enlentecimiento del habla y la dificultad para mantener el tema del discurso. La labor de la intervención logopédica especializada es crucial como tratamiento eficaz en el control de estos síntomas lingüísticos. Se defiende la necesidad de realizar una intervención logopédica acorde a las características y necesidades de la población objeto de estudio tomando como referencia la función metalingüística del lenguaje.

Palabras clave: personas mayores; deterioro cognitivo leve; demencia; intervención logopédica; aspectos lingüísticos; función metalingüística.

\section{[en] Linguistic manifestations in the elderly: the role of speech and language intervention in healthy and pathological aging}

\begin{abstract}
It has recently become particularly important to gain more knowledge about the linguistic and communicative abilities of the population who are over 65 years of age. The biological, psychological and social changes that this group of people suffers should be addressed taking into account the interaction between the cognitive and linguistic domains. With this in mind, the objective of this paper is to provide a literature review of linguistic manifestations, covering both common and pathological aging. Results show that the most outstanding features are mainly related to the difficulty of accessing the lexical label of words, the slowness of speech and the difficulty in maintaining the topic of the speech. Specialized-speech and language-therapy intervention (logopedic) is crucial to control and address these linguistic symptoms. It is for this reason that the need for speech and language-therapy intervention is highlighted. This intervention should be done by focusing on the specific characteristics and needs of aged people, taking as a point of departure the metalinguistic function of language.
\end{abstract}

Logopeda. Profesor Asociado de la Universidad de Valladolid. Departamento de Pedagogía.

enrique.gonzalez.martin@uva.es

2 Profesora Titular de Universidad. Departamento de Lengua Española. Área de Lingüística general. Universidad de Valladolid.

3 Profesora Titular de Universidad. Área de Psiquiatría. Universidad de Valladolid.

4 Logopeda. Profesora Asociada de la Universidad de Valladolid. Departamento de Pedagogía. 
Key words: elderly; mild cognitive impairment; dementia; logopedics; speech and language-therapy intervention; linguistic aspects; metalinguistic function.

Cómo citar: González Martín. E. et alii (2019). Manifestaciones lingüísticas en personas mayores: el papel de la intervención logopédica en el envejecimiento sano y patológico. Revista de Investigación en Logopedia, 9(1), 1-19.

\section{Introducción}

El Instituto Nacional de Estadística contempla que en el año 2018 España cuenta con más de 46 millones y medio de habitantes, de los cuales un 16,06\% (7.470.262) tienen 65 o más años. Ciertamente, a este grupo generacional se les incluye dentro del mismo parámetro poblacional, es decir, el de adultos mayores (Orosa Frais, 2003).

Muchas son las modificaciones o alteraciones que se producen en las personas mayores, tanto en el ámbito biológico como psicológico, social y, no menos importante, el ámbito lingüístico (San Miguel et al., 2006). Como señalan Horno Chéliz y Cuetos Vega (2016):

“A pesar de que el estudio de los marcadores biológicos está dando buenos resultados y es un campo muy prometedor, parece necesario completar el estudio diagnóstico con análisis alternativos y ciertas alteraciones lingüísticas sutiles que muestran estos pacientes pueden ser uno de ellos". (p. 176).

Respecto al tema que nos ocupa, el rendimiento en funciones neuropsicológicas y cognitivas, el Manual de Clasificación de Trastornos Mentales de la Asociación Americana de Psiquiatría, o sistema DSM-5 (Asociación Americana de Psiquiatría, 2014), establece los siguientes dominios neurocognitivos: atención compleja, función ejecutiva, aprendizaje y memoria, habilidades perceptuales motoras, reconocimiento social -o cognición social- y lenguaje.

Además, la clasificación propuesta para estos trastornos neurocognitivos (Asociación Americana de Psiquiatría, 2014) permite básicamente diferenciar las siguientes categorías de personas mayores, en función de su rendimiento neurocognitivo:

- Personas sanas (sin alteraciones cognitivas). Son aquellas personas cuyas características neurolingüísticas y cognitivas se justifican como cambios fisiológicos propios de la edad.

- Personas con Trastorno Neurocognitivo Leve o Deterioro Cognitivo Leve (en adelante DCL). Se trata de aquellas personas que presentan un declive cognitivo leve o moderado respecto al funcionamiento previo, que no interfiere en la capacidad de independencia en las actividades cotidianas, y que, por tanto, no afecta de forma significativa al funcionamiento global de la persona. Supone un estadio transitorio entre la normalidad, entendida como ausencia de patología, y la patología grave. En la versión previa de dicho manual de clasificación, el DSM-IV-TR (Asociación Americana de Psiquiatría, 2002), se denomina Trastorno Neurocognoscitivo Leve y no constituía propiamente un estado patológico. 
- Personas con Trastorno Neurocognitivo Grave o Demencia (en adelante DCG: Deterioro Cognitivo Grave). A diferencia del grupo anterior, existe un declive cognitivo significativo respecto al funcionamiento previo, que sí interfiere con la autonomía en las actividades cotidianas, y que, por tanto, afecta de forma intensa al funcionamiento global de la persona.

Dadas estas consideraciones sobre la clasificación propuesta, el entrenamiento en cualquiera de los dominios neurocognitivos mencionados podría favorecer la capacidad de independencia de las personas en las actividades cotidianas y, en consecuencia, su funcionamiento global en los diversos ámbitos (personal, laboral, social, comunicativo...), competencias que forman parte de la labor de profesionales de diversos ámbitos y en concreto de los logopedas, si atendemos, asimismo, a las habilidades comunicativas.

Por ello, y en lo que respecta al lenguaje, los usuarios que forman parte de las tres categorías citadas son considerados candidatos idóneos para formar parte de programas de carácter logopédico, con objetivos y características variables, pudiéndose impartir estos programas en entornos diversos, tales como ambulatorios, centros de día, centros geriátricos o de larga estancia, entre otros.

De esta manera, la intervención logopédica en personas mayores sanas constituye un elemento preventivo de posibles futuros problemas en el lenguaje y la comunicación, así como para aquella población con DCL para la que servirá de estimulación y elemento rehabilitador con el fin de preservar estas dos funciones; por todo ello, para las personas con demencia será un elemento fundamental en el intento de retrasar la evolución negativa de estos dos componentes tan imprescindibles en la vida cotidiana de cualquier persona. En resumen, para los tres grupos antes señalados (personas sanas, DCL y DCG) el objetivo final de la logopedia incluye la mejora del lenguaje y comunicación, y con ello, de la calidad de vida y el funcionamiento global de la persona.

\section{Lenguaje y comunicación en personas mayores sanas}

A continuación, destacamos las principales características encontradas en las personas mayores sanas, con DCL y con DCG o demencia, basándonos fundamentalmente en la comunicación y el lenguaje; no obstante, será indispensable hacer referencia a otros dominios cognitivos dada la continua interacción existente entre todos ellos.

Diversas investigaciones nos muestran que, a medida que envejecemos, algunas funciones lingüísticas se modifican. Según Labos, Del Río y Zabala (2009) "los efectos de la edad repercuten en tareas lingüísticas selectivas implicadas en el procesamiento léxico, sintáctico y discursivo" (p. 9). Estos déficits no se deben a un problema en el propio lenguaje, sino más bien a alteraciones en el ejecutivo central, especialmente en los procesos atencionales, en la memoria operativa y en la disminución de la rapidez en el procesamiento de la información (Juncos, Pereiro y Rodríguez, 2005; Juncos-Rabadán y Rozas, 2002).

En referencia a los parámetros lingüísticos que podemos identificar en la población sana mayor de 65 años, el conjunto de fenómenos en cada nivel de la lengua se manifiesta en los siguientes aspectos: 
En la competencia fonética-fonológica no se aprecian alteraciones en la persona sana ni en los primeros estadios del DCL (Bayles, Kazniak, Tomoeda, 1987), aunque sí en estadios de DCG o demencias.

Si atendemos a la competencia léxica, a pesar de que el léxico en las personas mayores tiende a mantenerse y/o incrementarse a través de la experiencia (Puyuelo y Bruna, 2006; Verhaeghen, 2003), se han observado dificultades en el acceso al mismo (Juncos-Rabadán, Facal, Álvarez y Rodríguez, 2006). Los cambios que se producen consisten en la ambigüedad para definir conceptos, reducción del número de sinónimos y aumento de las perífrasis verbales (Labos, Del Río y Zabala, 2009). Asimismo, se incrementa la frecuencia del fenómeno de la punta de la lengua (Labos, Del Río y Zabala, 2009; Puyuelo y Bruna, 2006), la incapacidad para evocar nombres de personas, así como el tiempo de reacción y el uso de circunloquios como estrategia compensatoria (Juncos-Rabadán y Rozas, 2002). Se observa una conservación del léxico pasivo como señalan en trabajos ya clásicos de Wingfiel, Aberdeen y Stine (1991); no obstante, en estudios previos se ha recogido un aumento de la dificultad para acceder a los nombres, o estadios muy frecuentes de DCL (Obler y Albert, 1984).

Por otra parte, en relación a la sintaxis, se producen problemas en la comprensión de oraciones (López-Higes y Rubio-Valdehita, 2014). Los estudios de Kemper (1989) añaden dificultades en la repetición y el uso espontáneo de oraciones complejas, debido a la disminución de la memoria de trabajo. En otros trabajos sobre sintaxis y población mayor, se ha demostrado que con la edad disminuye la longitud y la complejidad sintáctica de las oraciones (Salazar Provoste, 2007).

En cuanto al discurso, tanto oral como escrito, las personas mayores tienden a reducir el contenido de sus expresiones (Puyuelo y Bruna, 2006), perdiendo calidad en la estructuración y en el contenido discursivo. Tienen más dificultad en recordar y comprender el discurso del otro, debido fundamentalmente a problemas de memoria asociados a la edad (Juncos-Rabadán y Rozas, 2002) y a una menor eficacia a la hora de inhibir información irrelevante en la comprensión de textos. Esto da lugar a interpretaciones subjetivas de la información recibida (Puyuelo y Bruna, 2006). Asimismo, presentan dificultades cuando se les pide que cuenten una historia anteriormente relatada por otras personas, que resuman un texto o que deduzcan la resolución de una historia o moraleja. Según Bayles, Kazniak y Tomoeda (1987) se observan dificultades en el establecimiento de las referencias en el discurso, tanto en los recursos lingüísticos referenciales como los anafóricos y los deícticos.

Asimismo, han de considerarse también los efectos en la comunicación de la posible patología comórbida, como puede ser la presbiacusia (Valero, Bruna y Signo, 2012); así como el hecho de que algunos aspectos del lenguaje mejoran con la edad, como el vocabulario (Lojo-Seoane, Facal, Juncos-Rabadán y Pereiro, 2014).

Estudios recientes han introducido el concepto de reserva cognitiva para explicar la variabilidad en algunas dimensiones lingüísticas de las personas mayores. Rodríguez y Sánchez (2004) se refieren al término como "la capacidad de activación progresiva de redes neuronales en respuesta a demandas crecientes" (p. 6). Dicho de otro modo, se trata de un proceso normal del cerebro, tras la ejecución de tareas intelectuales. Cuantos más patrones complejos de actividad mental existan, menor es el riesgo de padecer demencia (López-Higes, Rubio-Valdehita, Prados y Galindo, 2013). Por ejemplo, se ha propuesto el bilingüismo como factor protector ante la demencia (Bak, Nissan, Allerhand y Dearvy, 2014; Calvo et al., 2016). Variables 
adquiridas como el nivel educativo, la escolaridad, la capacidad lectora, aficiones, el ejercicio físico y/o la alimentación influyen en la formación de la reserva cognitiva (Stern, 2006) y actúan como una compensación en el caso de que existiera una enfermedad neurodegenerativa (Rodríguez y Sánchez, 2004).

En relación a las habilidades lingüísticas, el estudio de López-Higes et al. (2013) indica que la escolaridad de la persona, la escolaridad de los padres, la ocupación laboral y la actividad lectora, se relacionan con el rendimiento en denominación, comprensión gramatical y vocabulario. Los años de escolaridad influyen en el rendimiento lector y el aumento de edad se asocia con el rendimiento en la denominación, que disminuye si descienden los años de escolaridad. En definitiva, una reserva cognitiva con buenas experiencias lectoras a lo largo de la vida, una ocupación activa $\mathrm{y}$ un buen rendimiento cognitivo son elementos fundamentales para combatir, o al menos retrasar, los déficits en la memoria operativa.

\section{El lenguaje y la comunicación de las personas mayores con deterioro cognitivo leve}

Como se ha indicado previamente, el DCL se concibe como un estadio de transición situado entre el envejecimiento normal y la demencia, que agrupa a pacientes con un declive cognitivo superior al observado en el envejecimiento normal, si bien no se afecta de forma significativa la autonomía ni el funcionamiento global de la persona. Los criterios diagnósticos del DCL que han causado mayor impacto y han tenido mayor seguimiento han sido los de Petersen (2004), que han sido admitidos por la mayor parte de expertos. Se manifiesta generalmente mediante quejas de memoria, deterioro objetivo de la memoria o de otras funciones cognitivas, y mantenimiento de la realización de actividades de la vida diaria (Juncos-Rabadán et al., 2010; Sperling et al., 2011). Así pues, tales manifestaciones no son suficientes para el diagnóstico de demencia.

Hernández Jaramillo (2010) señala que los cambios en la comunicación son los primeros indicios del DCL y los trastornos lingüísticos constituyen una de las primeras manifestaciones de demencia en casos de Alzheimer (Subirana, Bruna, Puyuelo y Virgili, 2009). La fluidez verbal, la denominación y el aprendizaje de las palabras son los mejores indicadores para diagnosticar su aparición (Juncos-Rabadán, 2009; Juncos-Rabadán, Pereiro, Facal y Rodríguez, 2010). Actualmente, la memoria verbal sería la principal dimensión lingüística que debería evaluarse, según una revisión de los principales estudios experimentales sobre lenguaje y DCL realizada por Juncos-Rabadán et al. (2010).

En la actualidad existen resultados contradictorios respecto a la presencia del fenómeno de la punta de la lengua (PDL) en personas con DCL. Mientras que para Van Der Hiele y cols. (2007), en su estudio sobre la correlación entre las medidas del electroencefalograma (EEG) y las pruebas cognitivas no hallaron diferencias significativas con los ancianos sanos en la prueba de vocabulario, otros confirman que las personas con DCL obtienen menor puntuación en vocabulario, denominación (parafasias) y tienen menor información semántica (Rodríguez, Juncos-Rabadán y Facal, 2008). La sintaxis y el discurso son los niveles menos estudiados en este tipo de población, sin embargo, en concreto el discurso narrativo ha sido objeto de estudio en 
algunas investigaciones actuales (Salazar Provoste, 2007). Así pues, se ha observado la existencia de alteraciones en el habla y fluidez del discurso, frases más breves y menor rendimiento en el relato autobiográfico (Chapman et al., 2002). Un estudio sobre el discurso con elicitación mediante imágenes, concluye que las personas con DCL presentan menor rendimiento discursivo con diferencias estadísticas respecto al grupo control (Alonso-Sánchez, Solis, Miranda y Mardones, 2018). Además, una de las áreas de la prueba que medía la coherencia local y el uso excesivo de pronombres y referentes inespecíficos, se puede atribuir a dificultades en el nivel sintáctico del sujeto (Alonso-Sánchez et al. 2018). En este orden de cosas, Shekim y La Pointe (1984) estudiaron la habilidad de participantes con demencia para producir discursos narrativos y observaron que hacían significativamente más referencias exofóricas, más desviaciones de oraciones, más pausas superiores a cinco segundos y utilizaban pocos recursos cohesivos.

Conocer y precisar estas variaciones lingüísticas que se producen en la persona en la fase DCL implica un mayor acceso al diagnóstico diferencial con respecto a la persona sin deterioro. Estos signos no son tomados en cuenta por familiares o proveedores de salud, que se limitan a esperar la aparición de cambios en las actividades de la vida diaria, o las propias señales indicativas de deterioro son consideradas dentro del patrón normal del envejecimiento (Tubero, 1999). Como indican Rodríguez et al. (2008), el diagnóstico diferencial entre DCL y el proceso demencial se realiza por parámetros de funcionalidad y no por criterios diagnósticos lingüísticos más específicos en cada una de las fases del deterioro, hecho que ayudaría a tener un diagnóstico y tratamiento más temprano y, por tanto, más eficaz.

\section{El lenguaje y la comunicación de personas mayores con deterioro cognitivo grave o demencia}

Siendo el lenguaje una de las funciones cognitivas que más tardan en sufrir alteraciones por el envejecimiento o el DCL, en las demencias ocurre a la inversa, los primeros síntomas están relacionados con un trastorno lingüístico (Subirana, Bruna, Puyuelo y Virgili, 2009). El deterioro del lenguaje de las personas mayores con demencias se inicia con una alteración en la función pragmática del lenguaje. Dentro de estas funciones destacan aspectos como la toma de turno conversacional y la máxima del principio de cooperación relativa a la relación o relevancia del tema de conversación. Posteriormente, estas personas se ven en mayor medida afectados en los aspectos semánticos de la lengua que se relacionan con los significados de las palabras. Por último, la patología extiende las dificultades lingüísticas al componente sintáctico y, en etapas posteriores más tardías, provoca déficits en la fonología, llegando, en el último estadio, al mutismo total (Cuetos, Arango-Lasprilla, Uribe, Valencia y Lopera, 2007).

Dentro de las demencias en población envejecida, la enfermedad de Alzheimer (EA) es una patología de tipo neurodegenerativa que se caracteriza por la pérdida progresiva de las funciones cognitivas (memoria, cálculo, orientación espacial y temporal, razonamiento, etc.) como consecuencia de alteraciones neuropatológicas que afectan a la corteza cerebral, fundamentalmente a las áreas temporoparietales (Horno y Cuetos 2016). 
En los casos de Alzheimer podemos distinguir una evolución temporal que abarca seis fases organizadas en tres estadios:

1. Estadio trasentorrinal (fases I y II): esta primera fase de la enfermedad se caracteriza porque la patología neurofibrilar se concentra en el córtex entorrinal y trasentorrinal, con ligera alteración del hipocampo.

2. Estadio límbico (fases III y IV): aparecen los primeros síntomas visibles y la alteración se extiende a otras áreas (entorrinales, hipocampo, amígdala, tálamo, hipotálamo y ganglios basales).

3. Estadio neocortical (fases V y VI): es la última fase de la enfermedad, ya completamente visible, con abundante patología neurofibrilar en todo el neocórtex y un grave deterioro cognitivo (Horno y Cuetos, 2016).

Como señalan los autores citados, "lo deseable sería poder detectar la enfermedad en el estadio primero, antes de que aparezcan los síntomas clínicos" (Horno y Cuetos, 2016, p. 176). Además, muchos síntomas lingüísticos prematuros son un claro indicador de algún tipo de demencia posterior.

La evolución del deterioro de la capacidad comunicativa de un paciente con esta patología se puede constatar a la vista de la siguiente tabla:

Tabla 1: Modelo de pérdida de las habilidades lingüísticas en la enfermedad de Alzheimer (Salazar Provoste, 2007, p. 56).

\begin{tabular}{|l|l|}
\hline Estado de evolución de la EA & Habilidad comunicativa afectada \\
\hline Leve & Competencia pragmática \\
\hline Media & Competencia semántica \\
\hline Moderada & Competencia sintáctica \\
\hline Severa & Competencia fonológica \\
\hline Estado final & Mutismo \\
\hline
\end{tabular}

Según esta tabla, la alteración del lenguaje en esta patología avanza desde el contenido, es decir, la pragmática y la semántica, hacia la forma, la sintaxis y la fonología. Por ello, es importante profundizar en el estudio de la pragmática y los marcadores discursivos para diagnosticar de forma precoz esta enfermedad (Salazar-Provoste, 2007). Las investigaciones sobre la producción del discurso en la enfermedad de Alzheimer desde el punto de vista macrolingüístico, señalan que estas personas emiten información irrelevante y son incapaces de establecer conexiones entre las unidades del discurso (Bschor, Kühl y Reischies, 2001; Ska y Duong, 2005). En el estudio de Toledo el al. (2018) sobre la identificación de las alteraciones de los aspectos macrolingüísticos del discurso, utilizando para ello la herramienta informática Coh-Metrix de análisis de textos, se comprobó una mayor dificultad en la planificación y organización de las ideas relacionadas con el tema; oraciones vacías con menos información y problemas de coherencia global. A esto se añaden las dificultades para mantenerse en el contexto discursivo y ajustarse a los cambios de turno con el interlocutor, lo que dificulta el seguimiento de la conversación y, en definitiva, la comprensión entre los interlocutores (Valles, 2013). Las pausas discursivas de estos pacientes también podrían ser utilizadas como parámetros de diagnóstico para detectar los déficits lingüísticos pues, según se pudo comprobar, su discurso es más pausado y menos fluido por las dificultades 
que tienen para encontrar las formas lingüísticas adecuadas (Rodríguez, Martínez y Valles, 2015).

Realmente, las personas con enfermedad de Alzheimer, lingüísticamente cometen muchos errores, que tienden a aumentar con el avance de la enfermedad. Además, los tipos de errores van cambiando en los diferentes estadios de la enfermedad que pueden comenzar, de acuerdo con Rumbo y Valles (2010), con problemas para evocar la información y seguir una conversación normal con las personas con las que se relacionan en su vida diaria. Pasan después por errores semánticos, al cambiar un nombre por otro similar (parafasias fonológicas, semánticas o inconexas) que se relacionan con la memoria semántica y la degradación del almacenaje de los significados de las palabras (Rodríguez-Ferreiro, Coll-Florit, Andreu y Sanz-Torrent, 2012); errores visuales: cambian un nombre por otro que tiene un referente visual muy parecido (pelota por naranja); realizan muchos circunloquios, dando la definición o el uso de un referente o un concepto; no responden a lo que se les demanda o caen en constantes neologismos como consecuencia de las parafasias inconexas. En trabajos como el de Cuetos, González-Nosti y Martínez (2005) se encuentran casos de errores que van aumentando en frecuencia dependiendo del estadio en el que se encuentren los pacientes y la etiología de la demencia (Valles, 2013).

\section{Evaluación del lenguaje personas mayores}

Centrándonos en la evaluación de las personas mayores, desde el punto de vista estrictamente logopédico, son escasas las pruebas que pueden diagnosticar problemas del lenguaje y comunicación. Ciertamente existen pruebas que pretenden diagnosticar determinadas patologías concretas (afasia y síntomas afásicos) en personas que pueden cumplir el criterio de pertenecer a la tercera generación, como pueden ser el test de Boston (Godglass y Kaplan, 1986) o la batería BETA (Cuetos y González-Nosti, 2009). Una de sus principales características es que son muy amplias, costosas y largas de aplicar. Sin embargo, destacamos seis pruebas que sí podrían responder a nuestro objetivo concreto, la evaluación del lenguaje y comunicación en el anciano sano o con trastorno cognitivo, bien de forma aislada o combinadas entre sí:

- Neurobel: Breve batería neuropsicológica de evaluación del lenguaje oral en adultos-mayores (Adrián, Jorquera y Cuetos, 2015). Una de sus características fundamentales es que actualmente sólo está comprobada su validez y fiabilidad en personas adultas en fases preclínicas, es decir, incluyendo sólo a los adultos sanos o aquellos con DCL. Consta de 8 subpruebas o tareas, 4 de ellas valoran la vertiente receptiva o de comprensión del lenguaje y las cuatro restantes la expresiva o de producción: A. Comprensión (1. Tarea de discriminación de fonemas, 2. Tarea de decisión léxica auditiva, 3 . Tarea de emparejamiento palabra hablada-dibujo, 4. Tarea de comprensión de oraciones). B. Producción (1. Tarea de repetición, 2. Tarea de denominación de dibujos, 3. Tarea de denominación de acciones, 4. Tarea de completar oraciones).

- Test Ecco_Senior (López-Higes, Rubio-Valdehita, Martín-Aragoneses, Del Río y Mejuto, 2012). Se basa en la evaluación de la comprensión del lenguaje escrito en personas mayores. Es la versión reducida y actualizada de la batería 
original (ECCO) para adultos mayores y pretende el estudio del perfil lingüístico de pacientes con DCL.

- Test de denominación de Boston (Goodglass y Kaplan, 1986). Constituye un test imprescindible para el estudio de la memoria semántica en los protocolos de evaluación de las demencias. Es una de las pruebas más frecuentemente utilizadas para evaluar la denominación en personas con enfermedad de Alzheimer. Debido a su longitud, se han desarrollado varias formas abreviadas para facilitar su aplicación. La versión original consta de 60 figuras de objetos, que han de denominarse por orden creciente de dificultad (Serrano et al., 2001).

- Token Test (De Renzi y Vignolo, 1962). Permite examinar la comprensión del lenguaje oral. El material consiste en 20 fichas de distintas formas, colores y tamaños. La finalidad de este test es evaluar la capacidad del paciente para comprender el nombre (círculo y cuadrado, color y tamaño), los verbos y las preposiciones incluidos en las instrucciones. De Renzi y Faglioni en 1978 elaboraron una versión reducida de la prueba idónea para personas mayores, basada en 36 consignas orales ordenadas por nivel de complejidad creciente.

- Protocolo de Exploración de Habilidades Metalingüísticas Naturales en la Afasia. MetAphAs (Rosell Clari y Hernández Sacristán, 2014). Su objetivo es valorar la capacidad de acceso al lenguaje interior y la inhibición del proceso de externalización del lenguaje oral. Los resultados muestran perfiles de afectación de pacientes con diferente tipo y severidad de la afasia, si bien, también es útil para evaluar las habilidades metalingüísticas de las personas con demencia (Valles y Rosell Clarí, 2016).

- PREP-R. Protocolo Rápido de Evaluación Pragmática Revisado (Fernández-Urquiza, Díaz, Moreno, Lázaro y Simón (2015). Es un instrumento screening que evalúa la eficacia pragmática y detecta los aspectos pragmáticos y/o componentes gramaticales en personas con déficit comunicativo.

Aparte de las anteriores, un tipo de pruebas que pueden resultar de gran utilidad para realizar un diagnóstico precoz de la enfermedad de Alzheimer o de cualquier demencia en población envejecida, es el grupo de test que implica el procesamiento léxico-semántico, puesto que el déficit lingüístico asociado a esta enfermedad está fundamentalmente relacionado con la memoria semántica. Como señalan estos autores, las tareas más relevantes para el diagnóstico temprano se encuadran en aspectos que evalúan la denominación oral de imágenes, la fluidez verbal semántica, la asociación semántica de imágenes, la clasificación de imágenes y el emparejamiento de imágenes con palabras.

Es evidente que hay una correlación entre la fase de la enfermedad (estado trasentorrinal, estado límbico y estadio neocortical) y el tipo de imágenes que los pacientes encuentran más difíciles de nombrar. Como señalan Cuetos, Rodríguez-Ferreiro, Sage y Ellis (2012), las personas van perdiendo la capacidad de nombrar objetos cuyos nombres han aprendido a una edad tardía de su vida, y este síntoma se aprecia más cuanto más va avanzando la enfermedad. Sin embargo, los mismos estudios indican que estos participantes conservan las palabras que han aprendido en una edad temprana y las últimas palabras que aprenden a lo largo de su vida son las que primero desaparecerán de su mente ${ }^{5}$. 
Muy interesante resulta el trabajo de Cuetos, Menéndez y Calatayud (2007) quienes han intentado elaborar una prueba de diagnóstico precoz de la enfermedad de Alzheimer. Para ello reutilizan algunos de estos test y añaden otros aspectos como la denominación por definición, denominación de nombres propios, comprobar a partir de una lista de palabras el recuerdo inmediato (o demorado), así como de la información sobre una persona, comprobar el recuerdo inmediato o demorado (Horno Chéliz y Cuetos Vega, 2016).

Las pruebas de evaluación del lenguaje de personas mayores están enfocadas principalmente en los aspectos semánticos y léxicos de la lengua y en aspectos fonológicos en menor medida (decir palabras que comiencen por un determinado fonema; decir nombres propios de personajes famosos, etc.).

Es habitual encontrar pruebas de fluidez verbal, como nombrar todos los referentes de una determinada categoría (tanto de elementos naturales como artificiales); pruebas de asociación semántica de imágenes (elegir, entre dos imágenes situadas en la parte inferior de una página, la que esté más relacionada con la imagen de la parte superior); pruebas orales y escritas de denominación de imágenes; clasificar imágenes en categorías semánticas; emparejar imágenes con palabras oídas o leídas; lectura de pseudopalabras; pruebas de denominación por definición; denominación de nombres propios; pruebas de memoria inmediata o demorada, etc.

Todas estas pruebas son fundamentales para el diagnóstico y la evaluación precoz de enfermedades como las que nos ocupan en personas mayores. No obstante, si atendemos a la tabla 1, observamos que no debemos dejar pasar por alto el componente pragmático, el uso de la lengua en contextos, la interpretación del lenguaje en emisiones o en interacciones comunicativas. Contar con pruebas de diagnóstico y evaluación que integren aspectos pragmáticos de la lengua, puede resultar un complemento indispensable para una completa evaluación de estas personas. Por ello, en este trabajo defendemos la creación de materiales, tanto pruebas de diagnóstico y evaluación como recursos de intervención logopédica que incluyan, aparte de aspectos de niveles fonológicos y léxico-semánticos, elementos metalingüísticos propios de la propia reflexión consciente que la persona hace de su lengua. Defendemos, pues, que en las personas mayores sanas o con deterioro cognitivo leve, se intervenga con materiales ad hoc que aprovechen el propio conocimiento que el hablante tiene de su lengua (función metalingüística del lenguaje) para un tratamiento o mantenimiento lingüístico más eficaz.

Estudios como el de Valles (2011) adelantan aspectos que nos parecen imprescindibles para una correcta intervención aprovechando los recursos metalingüísticos de la propia persona: el análisis conversacional para la evaluación del paciente con DCL y todos aquellos elementos lingüísticos que sea necesario dominar en el transcurso de una conversación.

La concepción tradicional de las intervenciones logopédicas parte de la realización de una evaluación inicial para determinar el nivel de partida del participante. Tras esta, se realiza una intervención logopédica con el objeto de solucionar los déficits observados. A lo largo del proceso terapéutico se va realizando la llamada

al., 2012) pero también la frecuencia con que se usan las palabras o lo familiares que resulten (Cuetos, Rosci, Laiacona, Capitani, 2008, p. 249-260). También se ha estudiado que no parece relevante la variable categoría semántica ni longitud de la palabra, como señalan Boulenger, Mechtouff, Thobois, Brousselle, Jeanneroud y Nazir, 2008. 
evaluación procesual que nos sirve para ir ajustando la intervención e ir observando cómo evoluciona el paciente. Al terminar el proceso, se realiza una evaluación final para determinar si el paciente ha conseguido o no los objetivos propuestos en la partida. Pues bien, entendemos la realización de una evaluación inicial en las personas mayores para determinar si existen o no déficits comunicativos y lingüísticos. De no existir, se plantearía una intervención preventiva para intentar mantener el mayor tiempo posible las funciones preservadas. De existir déficits, se ejecutaría una intervención terapéutica con el objetivo de que los mismos no avancen a lo largo del tiempo. En cualquiera de los dos procesos, el logopeda realizará una evaluación procesual; no obstante, la prueba que nunca se llevará a cabo en esta población será la evaluación final.

Como decimos, es interesante orientar la intervención logopédica atendiendo al metalenguaje de la persona. Entendemos, de este modo, que el discurso metalingüístico tiene que ver con un decir del lenguaje, formando parte de este discurso metalingüístico aquellos enunciados en los que se dice algo del lenguaje o de cualquiera de sus aspectos. Siguiendo a González y Loureda (2001-2002) "hay un metalenguaje que es fruto del hacer con el lenguaje. En él se incluyen todos los fenómenos que pertenecen, pues, al lenguaje, las lenguas y los textos como tales" (p. 270). Esta es la concepción amplia que proponemos del metalenguaje en las intervenciones logopédicas, las cuales deberán formar parte integral del proceso rehabilitador del lenguaje, junto con las categorías metalingüísticas propuestas por Ivern y Perinat (2013), que a la vez guiarán una intervención logopédica basada en categorías metalingüísticas (Tabla 2):

Tabla 2. Categorías metalingüísticas. (Ivern y Perinat, 2013, p.165)

\begin{tabular}{|l|l|}
\hline \multirow{2}{*}{ Ajuste comunicativo } & $\begin{array}{l}\text { Corrección. } \\
\text { Autocorrección (espontánea e inducida). } \\
\text { Comprobación de la efectividad comunicativa. } \\
\text { Imitación. } \\
\text { Ajuste del discurso al nivel lingüístico del interlocutor. }\end{array}$ \\
\hline $\begin{array}{l}\text { Análisis del valor informativo } \\
\text { de los mensajes. }\end{array}$ & $\begin{array}{l}\text { Ampliación de la información (espontánea e inducida). } \\
\text { Detección de ambigüedades e incoherencias. }\end{array}$ \\
\hline Aspectos lúdicos del lenguaje. & $\begin{array}{l}\text { Lenguaje verbal que acompaña al juego. } \\
\text { Verbalización con intención lúdica o humorística. }\end{array}$ \\
\hline $\begin{array}{l}\text { Manifestaciones del conoci- } \\
\text { miento lingüístico. }\end{array}$ & $\begin{array}{l}\text { Regulaciones, sobreextensiones y otras. } \\
\text { Citas de enunciados, uso de verbos relacionados con la } \\
\text { comunicación. } \\
\text { Manifestación de conocimiento del lenguaje a nivel fo- } \\
\text { nológico, morfosintáctico, semántico y pragmático. } \\
\text { Manifestaciones del conocimiento del lenguaje escrito. }\end{array}$ \\
\hline $\begin{array}{l}\text { Manifestaciones del conoci- } \\
\text { miento lingüístico sobre otras } \\
\text { lenguas }\end{array}$ & $\begin{array}{l}\text { Interferencias lingüísticas. } \\
\text { Conocimiento de otras lenguas. }\end{array}$ \\
\hline
\end{tabular}




\section{Modelos de intervención logopédica en personas mayores}

\section{Intervención logopédica en personas mayores. Propuestas teóricas}

Existen multitud de Programas de Prevención e Intervención centrados tanto en el envejecimiento activo como en los procesos demenciales. Estos programas trabajan las diferentes funciones cognitivas con la finalidad de mejorar la calidad de vida de la persona mayor.

Desde la logopedia, la planificación de la intervención engloba tanto a ancianos sanos como a aquellos que padecen deterioro cognitivo o un diagnóstico de demencia (Juncos-Rabadán, 1993). Mantener, paliar y/o compensar sus capacidades lingüísticas son los objetivos fundamentales que el logopeda debe alcanzar durante la intervención con este colectivo. De igual forma, Juncos-Rabadán (1998) añade que se debe paliar y compensar el deterioro lingüístico, teniendo en cuenta los procesos atencionales, inhibidores y la organización de los procesos léxicos, sintácticos y discursivos.

El entrenamiento lingüístico se incluye dentro de las Terapias no Farmacológicas (TNFs) que se definen por Olazarán et al. (2010) como "Cualquier intervención no química, teóricamente sustentada, focalizada y replicable, realizada sobre el paciente o el cuidador y potencialmente capaz de obtener un beneficio relevante" (p.2). Una de las terapias es la Psicoestimulación Cognitiva, que trata de entrenar, estimular y/o activar todas las funciones cognitivas, entre ellas, el lenguaje (CRE Alzheimer, 2016). Desde la Asociación Americana de Habla, Lenguaje y Audición o ASHA (2015), el trabajo del logopeda incluye tratar aspectos cognitivos de la comunicación dañados por un proceso de demencia para lograr un nivel funcional comunicativo acorde con el proceso de la enfermedad.

Otros programas de entrenamiento y/o estimulación cognitiva como "Activemos la Mente" de la Obra Social La Caixa (Peña-Casanova, 1999), el Programa de Psicoestimulación Integral o PPI (Tárraga et al., 2006), los "Cuadernos de Estimulación Cognitiva para Adultos" (Sardinero Peña, 2010), el "Programa de Actuación Cognitiva Integral de Demencias (PACID)" (García y Carro, 2011), herramientas de software como el programa Gradior (Franco, Orihuela, Bueno y Conde 2002) o "SmartBrain" (Sistema Interactivo de Estimulación Cognitiva) (Educamigos, 2018) entre otros, comparten un patrón de actividades relacionadas con la función del lenguaje divididas en una serie de áreas: lenguaje automático, lenguaje espontáneo, denominación, evocación y repetición categorial, repetición verbal, lecto-escritura, razonamiento, abstracción y otras funciones verbales. La intervención en estas áreas no se limita a los casos de envejecimiento patológico, sino que muchas de las actuaciones son de carácter asistencial y se realizan de forma grupal, para el mantenimiento de la actividad comunicativa y lingüística en la vejez normal (Juncos-Rabadán, 1993). La representación fonológica de la palabra o el fenómeno de la punta de la lengua (FPL) como se conoce habitualmente, ha sido profundamente estudiado con el propósito de averiguar las mejores estrategias para resolver con éxito las situaciones que implican FPL. Trabajar la estimulación del acceso al léxico a través de un procesamiento consciente y sin límite de tiempo, con abundantes claves semánticas, ayuda a activar las conexiones para el acceso a la forma fonológica de la palabra (Álvarez, Juncos-Rabadán, Facal y Pereiro, 2005). De forma complementaria, se han observado 
mayores efectos en la utilización de medidas de tipo discursivas, adaptadas al patrón lingüístico de las personas mayores sanas (Facal et al., 2009).

Por otra parte, existen estudios clínicos que han aplicado programas específicos del lenguaje centrados en las demencias según las necesidades, las características y el estado de gravedad de la persona. Los niveles léxico-semántico y pragmático han sido objeto de intervención principal ya que, como hemos indicado anteriormente, los primeros síntomas que desencadena el deterioro cognitivo tienen que ver con la pérdida de vocabulario y su uso (semántica y pragmática). Cullell, Bruna y Puyuelo (2006) están de acuerdo en implantar un tratamiento de logopedia para optimizar la preservación y la actividad funcional del lenguaje, basado en actividades de evocación, conciencia fonológica, trabajo con el discurso, etc., incorporando paralelamente una intervención neuropsicológica que incluya el trabajo con el resto de funciones cognitivas, "una intervención de carácter multidisciplinar que contemple la intervención psicoestimulativa como parte fundamental del tratamiento del Alzheimer" (Cullell, Bruna y Puyuelo, 2006, p. 237). Concretamente, el procesamiento de la información y la memoria son interdependientes, por ello una intervención logopédica debe ser integral, incluyendo la comunicación, el lenguaje y la memoria (Molero, 2001).

Otras técnicas como el Spaced Retrievel Training (SRT) de Landauer y Bjork (1976) y Schacter, Rich y Stamp (1985) (citados en Dober, 2016), son interesantes para esta propuesta pues intervienen en la memoria semántica, procedimental y prospectiva. Asimismo, los álbumes de recuerdo o los libros de memoria que son utilizados para la comunicación verbal (Bourgeois, 1990) y la intervención basada en los métodos Montessori, resultan exitosas entre personas con demencia y han sido utilizadas también en el campo de la logopedia con resultados muy satisfactorios (Mahendra et al. 2006; 2011).

Desde la función metalingüística del lenguaje, se ha comenzado a introducir programas logopédicos para las personas con demencia. Concretamente, Valles y Rosell-Clari (2016) han desarrollado un programa basado en estimular las habilidades metalingüísticas correspondientes a la Teoría de la Mente en contextos naturales a través de la comprensión y expresión tanto oral como escrita, con el fin de que los participantes mejoraran su comunicación con los interlocutores más cercanos, como vemos en la tabla 3:

Tabla 3. Habilidades metalingüísticas en Teoría de la Mente. (Valles y Rosell-Clari, 2016, p.92).

\begin{tabular}{|l|}
\hline 1. Describir un objeto o situación no presente \\
\hline 2. Recordar eventos pasados recientes \\
\hline 3. Recordar eventos pasados remotos \\
\hline 4. Anticipar eventos futuros \\
\hline 5. Describir escenas \\
\hline 6. Habilidad para contradecir o encontrar antónimos \\
\hline 7. Leer emociones \\
\hline 8. Uso de lenguaje ficticio \\
\hline 9. Habilidad para mentir \\
\hline 10. Expresar sarcasmo \\
\hline
\end{tabular}


La incorporación de tareas en la evaluación como la construcción de textos, la comprensión de situaciones comunicativas intersubjetivas y anticipar eventos, describir y analizar contextos, detectar estados emocionales y comprender actos de habla indirectos, se refleja en resultados efectivos en las personas objeto de la muestra, que previamente habían sido evaluados con el test MetAphAS (Valles y Rosell-Clari, 2016).

\section{El metalenguaje como procedimiento lingüístico en la intervención logopédica de personas mayores}

La función metalingüística o el metalenguaje es un procedimiento lingüístico utilizado para referirse a otros elementos lingüísticos del mismo o diferente enunciado o texto, así como para describir, analizar o interpretar una lengua natural (Hernández Alonso, 1996). La conciencia metalingüística de los hablantes, de todos los hablantes desde que adquirimos nuestra lengua siendo niños, se manifiesta en el hablar sobre el hablar mismo, en el hablar sobre la lengua y en el hablar sobre el lenguaje. Se trata de establecer referencia a algún enunciado previo real o supuesto.

El ámbito del léxico es el que más ha desarrollado la función metalingüística. Hablamos de metalenguaje para referirnos a muchos términos con los que las lenguas distinguen formas de hablar (mentir, acusar, susurrar, declarar, decir o conversar). Como señala Loureda (2007) también tenemos en las lenguas palabras para expresar cualidades de las personas en tanto que hablantes (locuaz, bienhablado) o de los discursos (lapidario, conciso, sucinto) o de los modos de presentarse en los textos (explicación, charla, comentario, conversación, noticia, etc.), todos estos términos hacen referencia al propio lenguaje, por lo tanto, estamos dentro del metalenguaje.

Las referencias a lo metalingüístico también se dan en las unidades fraseológicas equivalentes a sintagmas y/o palabras (Loureda, 2007): hablar como un papagayo, hablar como un descosido, hablar por los codos, hablar en Román paladino, hablar cara a cara, decir a los cuatro vientos, etc.

Como vemos, el metalenguaje trata la dimensión reflexiva del lenguaje (Loureda, 2007):

Lenguaje sobre el lenguaje que a) o hablan del lenguaje (discursos sobre el lenguaje, b) o presentan distinciones en el ámbito real del lenguaje (léxico, fraseología), etc., c) o son trozos de lenguaje presentados en el texto sobre los que se habla (uso metalingüístico) d) o son signos lingüísticos que para ser comprendidos (...) necesitan la "objetivación” de otros signos (enunciados-eco) (p. 20).

Para la enunciación metalingüística se dispone de diversas fórmulas según el tipo de operación que realicemos: definiciones, explicaciones, identificaciones, y el modo discursivo utilizado (enunciación, diálogo...). Como señala Hernández Alonso (1996), en una conversación o diálogo pluriactancial el emisor puede añadir en un determinado momento fórmulas metalingüísticas como: para que me entiendas..., te lo explicaré de otro modo; quiero decir...; o bien requerir del interlocutor: ¿lo entiendes bien?, ¿te has enterado? ¿has comprendido? Todos ellos son segmentos metalingüísticos inquisitivos. También el interlocutor de un diálogo o conversación puede interrumpir un turno conversacional y preguntar ¿y eso qué quiere decir?, 
explícate mejor, ponme un ejemplo, etc., que incitan a una reformulación, explicación o aclaración. También, en estos casos tan usados, estamos hablando de función metalingüística o metalenguaje.

Estas son, únicamente, algunas fórmulas de comunicación metalingüística intratextual (tanta anafórica como catafórica ${ }^{6}$ ). Nuestra propuesta de intervención en población adulta mayor de 65 años, con patología o sin patología lingüística, consiste en elaborar, diseñar, adaptar materiales y recursos que integren este tipo de fórmulas de comunicación metalingüística y metacomunicativa intratextuales para lograr una intervención más adecuada al tipo de población que estamos atendiendo. Al menos se pretende que el hablante utilice el conocimiento metalingüístico natural en su propio beneficio comunicativo.

Por otro lado, las fórmulas de metacomunicación son actos metalingüísticos que reproducen total o parcial, directa o indirectamente, algún enunciado previo, o bien hacen referencia a algún elemento aparecido o que aparecerá en el contexto. Estas fórmulas configuran lo que llamamos el discurso referido (citas y enunciados reproducidos): referencia a enunciados ya aparecidos o como pre-anuncio de algo que se va a decir, como por ejemplo, les repito que..., les diré que...; "es como una retroalimentación del mensaje con unidades emitidas para enfatizar o insistir en aquello" (Hernández Alonso, 1996, p. 382). Se trata de fórmulas muy utilizadas en el habla cotidiana y que pueden servir eficazmente en las intervenciones logopédicas para hacer más consciente al hablante de su propio uso del lenguaje; es decir, de nuevo estamos aplicando la función metalingüística en las sesiones de intervención.

Teniendo estos aspectos en cuenta, diversos estudios (Defyor,1996; Domínguez, 1996; Rincón y Pérez, 2009) han demostrado la utilidad del trabajo del metalenguaje para la mejora de los procesos lingüísticos, más concretamente los procesos lecto-escritores en una población infantil, incluso en niños con alguna alteración del lenguaje como la dislexia (Rueda, Sánchez y González, 1990). Estos estudios se ocupan normalmente de trabajar el análisis de la palabra, es decir, se centran en el componente fonético-fonológico del metalenguaje para la intervención, pero no utilizan el resto de los aspectos del metalenguaje ni se basan en otras categorías metalingüísticas diferentes a las mencionadas. Reiterando esta idea, consideramos que el componente metalingüístico, utilizado con personas adultas mayores de 65 años, que tienen unas competencias y habilidades lingüísticas plenamente adquiridas y, en muchos casos, no perdidas, influye positivamente en la intervención logopédica, tanto como trabajo preventivo como reparador, como ya señaló Juncos-Rabadán (1993), quien desde ya hace muchos años justificaba la importancia de ayudar a las personas a recordar una palabra a partir de un apoyo fonológico u ortográfico.

Dado que las personas mayores, con o sin patología que afecte a la comunicación y al lenguaje, pueden presentar dificultades en todos los componentes lingüísticos, es posible que una intervención que incluya la reflexión metalingüística y/ o metacomunicativa constituya un aspecto innovador y útil para la prevención, rehabilitación y retraso del deterioro cognitivo. Por esta razón, en este trabajo se sugiere que los procedimientos y mecanismos metalingüísticos son estrategias de intervención logopédica muy óptimas para la población mayor, como decimos, por su capacidad para

Referencia anafórica hacia atrás, es decir, a elementos del texto o el discurso presentados previamente. Referencia catafórica, o hacia adelante, hacia aspectos del discurso todavía no enunciados y que se citarán a continuación en el texto. 
reflexionar sobre su propia lengua y sobre los procedimientos gramaticales y léxicos que conforman la lengua objeto.

Entendemos que en la intervención con personas mayores es esencial estimular, prevenir el deterioro de la competencia comunicativa o frenarlo, entendido este proceso metalingüístico en palabras de Juncos-Rabadán (1993) como:

El conjunto de reglas de interacción que un hablante domina para poder comunicarse, refiriéndose por tanto a reglas de interacción social por las cuales una persona usa (comprende y produce) sus capacidades lingüísticas en un contexto comunicativo concreto (seguir una conversación, mantener normas de cortesía en el lenguaje, hacer un discurso, etc.) (p.79-80).

La intervención con esta población tan específica no se puede considerar como un camino hacia la alfabetización, al logro de objetivos escolares, a la mejora de los componentes del lenguaje desde un punto de vista académico, ni a la consecución de unos objetivos lingüísticos de partida. Respecto a estos objetivos, sucede que si no se alcanzan, se considera al paciente no apto para logopedia, y si se alcanzan, se le da el alta. Tampoco entendemos la intervención logopédica que aquí desarrollamos como una actividad ocupacional ni vacía de sentido con afán de entretener al paciente, ni como una mera estimulación de sus habilidades comunicativas.

Por consiguiente, consideramos que a través de la estimulación y desarrollo de la competencia lingüística como "el conjunto de reglas fonológicas, sintácticas y léxico-semánticas propias de una lengua determinada" (Juncos-Rabadán, 1993, p.80), trabajada desde su faceta metalingüística, puede lograrse la mejoría global de la competencia comunicativa en estas personas, que será el objetivo último de nuestra intervención.

Consideramos interesante, asimismo, para la elaboración de materiales y recursos para la intervención con adultos mayores, según este procedimiento metalingüístico y metacomunicativo, acudir a la práctica de resolución de dificultades o perturbaciones de la comunicación; es decir, practicar con los pacientes el papel del interlocutor en las conversaciones, intentando que usen los elementos lingüísticos que sirven para aclarar la comprensión del mensaje mediante procedimientos metalingüístico; por ejemplo, cuando el oyente pregunta: ¿cómo dice?, ¿qué entiende usted por...?, ¿qué quiere decir con...? O cuando el emisor contesta: "he dicho que..., he querido dar a entender que..., se lo diré con otras palabras...:"; todas estas fórmulas, tan rutinarias para un hablante competente, se convierten en herramientas muy útiles para que el logopeda las utilice en su programa de intervención logopédica en personas mayores sin demencias o con DCL.

\section{Conclusiones}

La revisión bibliográfica realizada sobre las manifestaciones lingüísticas en personas mayores pone de manifiesto, en primer lugar, la necesidad de atender a una clasificación tripartita entre personas sanas, con DCL y con DCG o demencias, así como que el diagnóstico precoz de un deterioro cognitivo será fundamental para paliar las consecuencias negativas de una falta de intervención. 
En segundo lugar, los rasgos más sobresalientes se manifiestan principalmente en la dificultad de acceso a la etiqueta léxica de las palabras, el enlentecimiento del habla y la dificultad para mantener el tema del discurso. La labor de la intervención logopédica especializada es crucial como tratamiento eficaz en el control de estos síntomas lingüísticos.

En tercer lugar se concluye que la función metalingüística del lenguaje nos proporciona una visión de la intervención logopédica más amplia, pues tomamos como referencia los conocimientos lingüísticos de los hablantes nativos. Por ello, proponemos un análisis más exhaustivo de los mecanismos lingüísticos que ponemos en práctica en el día a día para, utilizando el código lingüístico, hablar del lenguaje mismo.

El metalenguaje, y dentro de este, la reproducción lingüística, hace referencia global a una lengua, a una parte de ella (texto), a su historia y evolución, a sus diversas realizaciones dialectales. Este procedimiento lo utilizamos para explicar la gramática de una lengua, cuando enseñamos la lengua propia o ajena, cuando comentamos un texto. El metalenguaje es, en definitiva, toda actividad de explicación, comentario, análisis, exégesis o interpretación. Trabajando esta función, a través, por ejemplo del uso de los verba dicendi (decir, pretender, defender, repetir, reproducir, opinar, sugerir, etc.) estamos ayudando a la persona a hacer un uso más reflexivo de la lengua, lo cual repercute en el reforzamiento del uso que hace de ella; favorecemos, asímismo, que el hablante no pierda esta competencia lingüística, practicando el cambio de personas en la reproducción del discurso, el cambio de formas verbales en la correlación temporal; trabajamos la función metalingüística utilizando el discurso indirecto encubierto, reproduciendo indirectamente un enunciado, asumido por el enunciador, añadiendo una determinada intención. Con estos contenidos estamos, además, reforzando el componente pragmático-discursivo de la persona mayor, adulto competente que debe preservar el lenguaje al llegar a su vejez.

Podemos señalar que existen manifestaciones de lo metalingüístico (Loureda, 2007) en los tres niveles fundamentales del lenguaje que proponía Coseriu (1991): universal, histórico e individual. Hay hechos metalingüísticos universales; hay hechos de metalengua (alojados en el léxico, en la gramática); y hay hechos de metahabla o metadiscursivos (en las citas-eco, en la ironía o en los contenidos de discursos en los que se habla sobre el lenguaje). Se trata, en definitiva, de distinguir entre el hacer con el lenguaje (todos los fenómenos reflexivos que manifiesta el lenguaje, las lenguas y los textos como capacidad) y el decir del lenguaje (las predicaciones que se hacen sobre lo lingüístico).

Si aplicamos esta distinción entre el hacer con el lenguaje y el decir con el lenguaje la intervención logopédica se entiende como una actuación sobre el uso metalingüístico del lenguaje primario, esto es, un hacer con el lenguaje a nivel universal, histórico e individual, que se debe manifestar en la gramática del uso metalingüístico, el metalenguaje en el texto, el léxico metalingüístico, la fraseología metalingüística, así como hechos, estrategias, procedimientos y mecanismos metadiscursivos (Loureda, 2007) que se aplicarán a intervenciones que trabajen aspectos lingüísticos como la reformulación de enunciados, la atenuación, la intensificación o la propia intertextualidad. Estos aspectos son recursos de uso común en el discurso oral o escrito de cualquier hablante.

Defendemos la idea recogida por González y Loureda (2001-2002) de que lo "metalingüístico constituye un amplio y heterogéneo dominio que cobra presencia 
en muchos actos de habla" (p. 269) y ese dominio del uso metalingüístico del habla corriente es el que queremos aplicar a las intervenciones logopédicas, como cuando empleamos los signos relacionándolos conscientemente con otros signos; es lo que hacemos cuando usamos el lenguaje con fines humorísticos, o como cuando en la configuración de los signos se imita la forma sonora de los significantes de otra lengua, en el caso de los chistes.

En el trabajo anteriormente mencionado (González y Loureda, 2001-2002) se ha desarrollado la idea de si "la gran cantidad de intuición y experiencia almacenada en los significados de contenido metalingüístico puede tener alguna utilidad para los otros campos relacionados con el lenguaje" (p. 277); en el caso de estos autores, la aplicación se hace a la enseñanza de la lengua. No obstante, consideramos que hasta la fecha no se han sabido aplicar, o al menos no se ha desarrollado en profundidad, las ventajas que ofrece el uso metalingüístico de la lengua para intervenir en aspectos lingüísticos del lenguaje en población mayor. En este aspecto es donde debemos profundizar.

\section{Bibiografía}

Adrián, J.A., Jorquera, J., y Cuetos, F. (2015). NEUROBEL: Breve batería neuropsicológica de evaluación del lenguaje oral en adultos-mayores. Datos normativos iniciales. Revista de Logopedia, Foniatría y Audiología, 35, 101 - 113.

Alonso, M.F., Solis, C., Miranda, E. y Mardones, C. (2018). Discurso narrativo en sujetos con deterioro cognitivo leve. Cuadernos de Neuropsicología, 12 (1), $42-49$.

Álvarez, M., Juncos-Rabadán, O., Facal, D., y Pereiro, A. (2005). Efectos del envejecimiento en el fenómeno de la punta de la lengua. Sugerencias para la intervención en el acceso al léxico. Revista de Logopedia, Foniatría y Audiología, 25(3), 115-120.

American Speech and Language Hearing Association. (2015). Dementia: Resources. Recuperado de: http://www.asha.org/PRPSpecificTopic.aspx?folderid=8589935289\&section=Resources

Asociación Americana de Psiquiatría (2014). Manual Diagnóstico y Estadístico de los Trastornos Mentales DSM-5. Madrid: Editorial Médica Panamericana.

Asociación Americana de Psiquiatría (2002). Manual Diagnóstico y Estadístico de los Trastornos Mentales DSM-IV-TR. Barcelona: Masson

Bak, T. H., Nissan, J. J., Allerhand, M. M., y Deary, I. (2014). Does bilingualism influence cognitive aging? Annals of Neurology, 75, 959-963.

Bschor, T., Kühl, K.P. y Reischies, F.M. (2001). Spontaneous speech of patients with dementia of the Alzheimer type and mild cognitive impairment. International Psychogeriatrics, 13(3), 289-298.

Bayles K, Kazniak A.W., y Tomoeda, C.K. (1987). Communication and Cognition in normal aging and dementia. Boston MA: College- Hill, Press /Litte, Brown and Co.

Boulenger, V., Mechtouff, S., Thobois, E., Brousselle, M., Jeanneroud, T., y Nazir, A. (2008). Word Processing in Parkinson's disease is impaired for action verbs but not for concrete nouns. Neuropsychologia, 46, 743-756.

Bourgeois, M. (1990). Enhancing conversation skills in Alzheimer's disease using a prosthetic memory aid. Journal of Applied Behavior Analysis, 23, 29-42. 
Calvo, N., Manoiloff, L., Muñoz, E., Contreras, M., Ibáñez, A., y García, A.D. (2016). El bilingüismo como protección ante la demencia: consistencias empíricas y nuevas propuestas metodológicas. Círculo de Lingüística Aplicada a la Comunicación, 68, 3-44.

Chapman, S.B., Zientz, J., Weiner, M., Rosenberg, R., Frawley, W., y Burns, M.H. (2002) Discourse changes in early Alzheimer disease, mild cognitive impairment, and normal aging. Alzheimer disease and associated disorders, 16(3), 177-86.

Coseriu, E. (1991). El hombre y su lenguaje. (2a ed). Madrid: Gredos.

CRE Alzheimer (2016). Programas de psicoestimulación. Instituto de Mayores y Servicios Sociales. Recuperado de http://www.crealzheimer.es/crealzheimer_01/servicios_atencion_directa/promocion_autonomia_personal/rehabilitacion_funcional_neuropsicologica/programas_psicoestimulacion/index.htm

Cuetos, F., González-Nosti, M., y Martínez, C. (2005). The picture-naming task in the analysis of cognitive deterioration in Alzheimer's disease. Cognitive Brain Research, 17, 549-561.

Cuetos, F.J., Arango-Lasprilla,C., Uribe, C., Valencia, C., y Lopera, F. (2007). Linguistic changes in verbal expression: a preclinical marker of Alzheimer's disease. Journal of the International Neuropsychological Society, 13 (3), 433-439.

Cuetos, F., Menéndez, M., y Calatayud, M.T. (2007). Descripción de un nuevo test para la detección precoz de la enfermedad de Alzheimer. Revista de Neurología, 44, 469-474.

Cuetos, F., Rosci, C., Laiacona, M., y Capitani, E. (2008). Different variables predict a nomia in different subjects: a longitudinal study of two Alzheimer's patients. Neuropsychologia, 46, 249-260.

Cuetos, F., Rodríguez-Ferreiro, J., Sage, K., y Ellis, A. (2012). A fresh look at the predictors of naming accuracy and errors in Alzheimer's disease, Journal of Neuropsychologia, 46, 249-260.

Cuetos, F., y González-Nosti, M. (2009). Batería para la Evaluación de los Trastornos Afásicos (BETA). Madrid: Instituto de Orientación Psicológica EOS.

Cullell, N., Bruna, O., y Puyuelo, M. (2006). Intervención neuropsicológica y del lenguaje en la EA. Descripción de un caso clínico. Revista de Logopedia, Foniatría y Audiología, 38(1), 231-238.

De Renzi, E., y Vignolo, L. (1962). The Token Test; a sensitive test to detect receptive disturbances in aphasics. Brain, 85, $665-678$.

De Renzi E, y Faglioni P. (1978). Normative data and screening power of a shortened version of the Token Test. Cortex, 14, 41- 90.

Defyor, S. (1996). Una clasificación de las tareas utilizadas en la evaluación de las habilidades fonológicas y algunas ideas para su mejora. Infancia y Aprendizaje, 73, 49-63.

Dober, L. (2016). Memory support for Adults with Alzheimer's Disease: Applications for Speech-language Pathologists. Honors Theses. Western Michigan University.

Domínguez, A.B. (1996). Evaluación de los efectos a largo plazo de la enseñanza de habilidades de análisis fonológico en el aprendizaje de la lectura y de la escritura. Infancia y Aprendizaje, 76, 83-96.

Educamigos (2018). Smartbrain: sistema interactivio de Estimulación Cognitiva. Recuperado de https://www.smartbrain.net/smartbrainpro/

Facal, D., González, M.F., Buiza, C., Laskibar, I., Urdaneta, E., y Yanguas, J.J. (2009). Envejecimiento, deterioro cognitivo y lenguaje: Resultados del Estudio Longitudinal Donostia. Revista de Logopedia, Foniatría y Audiología, 1, 4-12.

Fernández-Urquiza, M., Díaz, F., Moreno, V., Lázaro, M., y Simón, T. (2015). PREP-R. Protocolo Rápido de Evaluación Pragmática Revisado. Valencia: Servicio de Publicaciones de la Universidad de Valencia. 
Franco-Martín, M. A., Orihuela, T., Bueno, Y., y Conde, R. (2000). Programa GRADIOR. Rehabilitación cognitiva por ordenador. Valladolid: Edintras.

García Meilán, J.J., y Carro Ramos, J. (2011). Programa de Actuación Cognitiva Integral de Demencias (PACID):Centro de Referencia Estatal de Atención a Personas con Enfermedad de Alzheimer y otras Demencias. Madrid: Instituto de Mayores y Servicios Sociales (IMSERSO).

González-Ruiz, R., y Loureda -Lamas, O. (2001-2002). Nuevos estudios sobre lo metalingüístico en español. Cuadernos de Investigación Filológica, 27, 28. 267-284.

Goodglass, H., y Kaplan, E (1986). La evaluación de la afasia y trastornos relacionados $\left(2^{a}\right.$ ed.) Madrid: Panamericana.

Hernández-Alonso, C. (1996). Procedimientos y mecanismos del metalenguaje en español. Lexis, 20(1-2), 373-392.

Hernández-Jaramillo, J. (2010). Demencias: los problemas de lenguaje como hallazgos tempranos. Acta Neurológica Colombiana, 26(3), 101-111.

Horno Chéliz, I. y Cuetos Vega, F. (2016). Manifestaciones lingüísticas tempranas de la enfermedad de Alzheimer. En M.C. Horno Chéliz, I. Ibarretxe Antuñano y J.L. Mendivil-Giró. Panorama actual de la ciencia del lenguaje (p. 175-191). Universidad de Zaragoza.

Ivern, I., y Perinat, A. (2013). La emergencia y evolución del metalenguaje en la infancia. Infancia y Aprendizaje, 36(2), 163-180.

Juncos-Rabadán, O. (1993). Consideraciones sobre el lenguaje y la intervención logopédica en la tercera edad. Revista de Logopedia, Foniatría y Audiología, 13(2), 79-88.

Juncos-Rabadán, O. (2009). Lenguaje en el deterioro cognitivo leve. Revista de Logopedia, Foniatría y Audiología, 29(1), 1-3.

Juncos-Rabadán, O., Facal, D., Álvarez, M., y Rodriguez, M. S. (2006). El fenómeno de la punta de la lengua en el proceso de envejecimiento. Psicothema, 18(3), 501-506.

Juncos-Rabadán, O., Pereiro, A.X., y Rodríguez, M.S. (2005). Narrative speech in aging: Quantitiy, information content and cohesion. Brain and Language, 95, 423-434.

Juncos-Rabadán, O., Pereiro, A.X., Facal, D., y Rodríguez, N. (2010). Una revisión de la investigación sobre el lenguaje en el DCL. Revista de Logopedia, Foniatría y Audiología, 2(30), 73-83.

Juncos-Rabadán, O., y Rozas, P. (2002). Problemas del lenguaje y la tercera edad. Orientaciones y perspectivas de la logopedia. Revista Galego-Portuguesa de Psicoloxía e Educación, (8), 387-398.

Labos, E., Del Río, M., y Zabala, K. (2009). Perfil de desempeño lingüístico en el adulto mayor. Revista Argentina de Psicología, 13, 1-13.

Lojo-Seoane, C. Facal, D., Juncos-Rabadán, O., Pereiro, A.X. (2014). El nivel de vocabulario como indicador de reserva cognitiva en la evaluación del deterioro cognitivo ligero. Anales de Psicología, 30(3), 1115-1121.

López-Higes, R., Rubio-Valdehita, S., Martín-Aragoneses, M.T., Del Río, D., y Mejuto, G. (2012). Evaluación de la comprensión gramatical en el envejecimiento normal y patológico: Un resumen de los resultados obtenidos con las baterías ECCO y ECCO_Senior. International Journal of Psychological Research, 5(1), 96-108.

López-Higes R., Rubio-Valdehita S., Prados J.M., y Galindo M. (2013). Reserva cognitiva y habilidades lingüísticas en mayores sanos. Revista de Neurología, 57, 97-102.

López-Higes, R., y Rubio-Valdehita, S. (2014). Variabilidad en la comprensión gramatical de mayores sanos: Diferencias en función de la reserva cognitiva. Revista de Logopedia, Foniatría y Audiología, 34(2), 51-59.

Loureda-Lamas, O. (2007). De la función metalingüística al metalenguaje: los estudios sobre el metalenguaje en la lingüística actual. En Cassol, A. Guarino, G, Mapelli, F, Matte Bon, 
P. Taravacci: Metalinguaggi e metastessi. Lingua, Letteratura e traduzione (p. 17-19). XXIV Congreso AISPI (Padova, 23-26 maggio 2007), Roma, AISPI Edizioni.

Mahendra, N., Hopper, T., Bayles, K.A., Azuma, T., Clearly, S. y Kim, E. (2006). Evidence-based practice recommendations for working with individuals with dementia: Montessori-based interventions. Journal of Medical Speech-Language Pathology, 14(1), 15-25.

Mahendra, N., Scullion, A., y Hamerschlag, C. (2011). Cognitive-linguistic interventions for persons with dementia: A practitioner's guide to 3 evidence-based techniques. Topics in Geriatric Rehabilitation, 27(4), 278-288.

Molero, R. (2001). Intervención logopédica en la edad adulta tardía: estimulación y mantenimiento cognitivo. Anales de Pedagogía, 19, 115-138.

Obler L.K., y Albert M.L. (1984). Clinical Neurology of Aging. New York: Oxford University Press.

Olazarán, J., Reisberg, B., Clare, L., Cruz, I., Peña-Casanova, J. Del Ser, T., et al., (2010). Eficacia de las terapias no farmacológicas en la enfermedad de Alzheimer: una revisión sistemática. Dementia and Geriatric Cognitive Disordes, 30, 161-178.

Orosa Frais, T. (2003). La tercera edad y la familia: una mirada desde el adulto mayor. La Habana: Editorial Félix Varela.

Peña-Casanova, J. (1999). Intervención cognitiva en la enfermedad de Alzheimer. Barcelona: Fundación "La Caixa".

Puyuelo, M., y Bruna, O. (2006). Envejecimiento y lenguaje. Revista de Logopedia, Foniatría y Audiología, 26(4), 171-173.

Rincón, M.L., y Pérez, J. (2009). Programa para el entrenamiento de la conciencia fonológica en niños de 5 a 7 años como prerrequisito para el aprendizaje de la lectura. Revista Areté, 9, 140-150.

Rodríguez, J., Martínez, H., y Valles, B. (2015). Las pausas en el discurso de individuos con demencia tipo Alzheimer. Estudio de casos. Revista de Investigación en Logopedia, 1, 40-59.

Rodríguez, N., Juncos-Rabadán, O., y Facal, D. (2008). El fenómeno de la punta de la lengua en el deterioro cognitivo leve. Un estudio piloto. Revista de Logopedia, Foniatría y Audiología, 28(1), 28-33.

Rodríguez, M., y Sánchez, J.L. (2004). Reserva cognitiva y demencia. Anales de Psicología, 20(2), 175-186.

Rodríguez-Ferreiro, J., Coll-Florit, M., Andreu, L. y Sanz-Torrent, M., (2012). L'afectació del llenguatge en la malaltia d'Alzheimer. LLengua, Societat Y I Comunicació, 10, 39-45. Recuperado de http://revistes.ub/index.php/LS

Rosell-Clari, V., y Hernández Sacristán, C. (2014). MetAphAs: Protocolo de Exploración de Habilidades Metalingüísticas Naturales en la Afasia. Valencia: Nau Llibres.

Rueda, M., Sánchez, E., y González, L. (1990). El análisis de la palabra como instrumento para la rehabilitación de la dislexia. Infancia y Aprendizaje, 49, 39-52.

San Miguel, A., Rodríguez Barbero, MJ., San Miguel, R., Alonso, N., Calvo, B., y Martín-Gil, FJ. (2006), Estudio de los marcadores biológicos en la enfermedad de Alzheimer. Revista Electrónica de Biomedicina 1, 88 - 99.

Salazar Provoste, O. (2007). Marcadores discursivos y discurso senescente: análisis del habla producida por ancianos normales y ancianos diagnosticados con demencia tipo Alzheimer. Tesis doctoral inédita. Universidad de Valladolid.

Sardinero Peña (2010). Estimulación cognitiva para adultos. Madrid: Grupo Gesfomedia.

Serrano, C., Allegri, R.F., Drake, M., Butman J., Harris P., Nagle, C., et al., (2001). Versión 
abreviada en español del test de denominación de Boston: su utilidad en el diagnóstico diferencial de la enfermedad de Alzheimer. Revista de Neurología, 33 (7), 624-627.

Shekim, L.O., y La Pointe, L.L. (1984), Production of dicourse in individuals with Alzheimer's disease. Paper presented at the 12 annual meeting of the International Neuropsychological Society, Houston, TX.

Ska, B., y Duong, A. (2005) Communication, discours et démence. Psychologie \& NeuroPsychiatrie du vieillissement, 3(2), 125-133.

Sperling, R. A., Aisen, P. S., Beckett, L. A., Bennett, D. A., Craft, S., Fagan, A. M., et al., (2011). Toward defining the preclinical stages of Alzheimer's disease: Recommendations from the National Institute on Aging-Alzheimer's Association workgroups on diagnostic guidelines for Alzheimer's disease. Alzheimer's \& Dementia: The Journal of the Alzheimer's Association, 7(3), 280-292.

Stern, Y. (2006). Cognitive reserve and Alzheimer disease. Alzheimer Disease \& Associated Disorders, 20(2), 112-117.

Subirana, J., Bruna, O., Puyuelo, M., y Virgili, C. (2009). Lenguaje y FFEE en la valoración inicial del DCL y la EA. Revista de Logopedia, Foniatría y Audiología, 29(1), 13-20.

Tárraga, L., Boada, M., Modinos, G., Espinosa, A., Diego, S., Morera, A., at el., (2006). A randomised pilot study to assess the efficacy of an interactive, multimedia tool of cognitive stimulation in Alzheimer's disease. Journal of Neurology, Neurosurgery, and Psychiatry, 77(10), 1116-1121.

Toledo, C. M., Aluísio, S. M., dos Santos, L. B., Brucki, S. M. D., Trés, E. S., de Oliveira, et al., (2018). Analysis of macrolinguistic aspects of narratives from individuals with Alzheimer's disease, mild cognitive impairment, and no cognitive impairment. Alzheimer's \& Dementia: Diagnosis, Assessment \& Disease Monitoring, 10, 31-40.

Tubero, A.L. (1999). A linguagem do envelhecer: Saúde e Doença. Disturbios da Comunicação, 10(2), 54-70.

Valero-García, J., Bruna Rabassa, O., y Signo, S. (2012). Envelliment i comunicació: interrelació entre els factors auditius, cognitius i emocionals. Revista de Psicologia, Ciències de l'Educació i de l'Esport, 30(1), 53-66.

Valles, B. (2011). Representaciones y creencias en torno a la evaluación lingüística del adulto mayor con trastorno cognitivo leve. Revista de Investigación en Logopedia 1 (1), 12-34.

Valles, B. (2013). Una aproximación al estudio de la conversación de la persona con Alzheimer y sus interlocutores sanos. Revista de Investigación en Logopedia, 3(2), 96-119.

Valles, B., y Rosell-Clari, V. (2016). Programa de Estimulación de las Habilidades Metalingüísticas en Teoría de la Mente (ToM) para personas con demencia: un estudio piloto. Lingua Americana, 38, 81-102.

Van der Hiele, K., Vein, A., Reijntjes, R., Westendorp, R., Bollen, E., et al. (2007). EEG correlates in the spectrum of cogni- tive decline. Clinical Neurophysiology, 118, 1931-1939.

Verhaeghen, P. (2003). Aging and vocabulary scores: a meta-analysis. Psychology and Aging, 18(2), 332-339.

Wingfield A., Aberdeen J.S., y Stine, EA. (1991). Word onset gating and linguistic context in spoken word and recognition by Young and elderly adults. The Journals of Gerontology: Psychological Sciences, 46, 127-129. 